\title{
Quantifying the LEctenna: Measuring the Invisible Made
}

\section{Visible}

\author{
Michelle L. Yu \\ Thomas S. Wootton High School, 2100 Wootton Pkwy, Rockville, MD, 20850, USA; michelle.1.yu13@gmail.com
}

\begin{abstract}
A light emitting diode (LED) rectifying antenna, also known as a LEctenna, is an easily constructible device (consisting only of an LED and a Schottky diode) that converts electromagnetic energy into direct current electricity. The LEctenna falls into the larger scope of power beaming and space solar, which is the process of wirelessly transmitting energy derived from sunlight in space to locations on Earth. More specifically, a satellite in space collects energy that the sunlight carries and beams the energy to Earth in the form of waves. A rectifying antenna on Earth collects the energy, converts it into direct current, and it is transported for consumption. The LEctenna serves as a representation of the rectifying antennas that are stationed on Earth. By implementing a self-developed brightness scale and a homemade invention that enhances radio frequency detection quantitative data was recorded with different LEctenna units. With this quantitative data, the overarching goal of this research is to develop conclusions centered around the behavior of the LEctennas in different situations as well as to analyze LEctennas in order to better understand everyday microwave energy.
\end{abstract}

KEYWORDS: Physics; Energy; Solar; Engineering; Power Beaming; Rectifying Antenna.

\section{- Introduction}

All around the world, millions of people lack electricity, making simple chores in daily life more difficult. Villages in Africa and the Middle East lack the proper resources to provide their residents with electricity. One possible solution is the use of space solar and power beaming. Space solar is the process of gathering energy from sunlight in space and wirelessly transmitting that energy to Earth. ${ }^{1}$ Energy from sunlight is abundant in space, as the sunlight is not obstructed or reflected by various objects. Satellites in space can collect the energy in sunlight and beam the energy to antennas stationed on Earth. Power beaming can bring electricity to even the most remote locations in the world, thus helping residents living in the country.

Another potential advantage of space solar power is that it opens a new realm of effectively unlimited energy. As fossil fuels continue to decline, the power via space solar can be a solution that offers safe and clean power to both the most populated cities and the most remote locations of the world. ${ }^{2}$ The LEctenna is a smaller representation of the rectenna that is stationed on Earth. The LEctenna uses the "invisible" microwaves to power a light that human eyes can detect. The research that was conducted explores this subcategory of the LEctenna. A brightness scale was developed and a device that allowed me to quantify the voltage and lux the LEctenna emitted, which had not previously been done before. This quantitative data helps to understand the properties of microwaves that are used in homes every single day and can be related to some aspects of power beaming.

\section{Methods}

\section{Making the LEctenna:}

When constructing the LEctenna, the black stripe on the
1N5711 Schottky diode was placed opposite the flat part of the LED, as seen in Figure 1 in a future section. It was recently found by a colleaque at the NRL that the NTE112 diode also works for the LEctenna.The black stripe represents the cathode side of the Schottky diode and the flat part of the LED represents its cathode side. The LEctenna performs best when the leads of the diode and LED are only wrapped around each other 2 to 4 times. The number of times the two leads are wrapped is relevant to the length of a microwave length. If the LEctenna's receivable wavelength is too long or too short compared to the microwave, the LEctenna will not light up to its full potential. Furthermore, the LEctenna is brighter if only one diode is paired with the LED, rather than pairing 2 or 3 diodes with an LED. Multiple diodes paired with one LED were tested, the results and more analysis can be found in Appendix B.

Additionally, a soldering iron was not used to join the two leads together. To clarify, the WiFi frequency was $2.4 \mathrm{G} \mathrm{Hz}$ or a dual band. A plastic straw was used to hold and stabilize the LEctenna as it was held up to objects. Other objects, such as a plastic test tube or glass straw, could also be used. One end of the LEctenna was taped to the end of the straw and it was held up to the Wi-Fi router. As long as one doesn't come in direct contact with the leads of the LEctenna, the straw can be held at any distance away from the LEctenna. When one touches the lead of the LEctenna, the resistance in the circuit increases and the light of the LED fades.

\section{Constructing the PARME Phone Attachable RF Meter}

Enhancer):

An important aspect when using the PARME is that the top of the LEctenna should be aimed towards the phone camera, 
seen in Figure 1, as viewed by the phone's camera. Additionally, when actually measuring the lux from the LEctenna, the device works best when the room is relatively dark, to avoid capturing extraneous light.

Figure 2 is a screenshot of the Lux Light Meter app opened on a phone. The application is free to use with a few helpful features, such as average and maximum lux. It should be mentioned that the numbers that are displayed in Figure 2 are

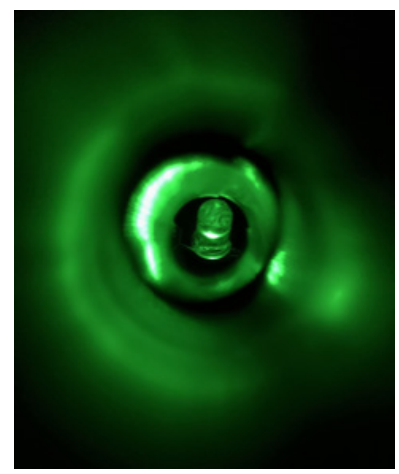

Figure 1: LEctenna inside of the shaft of the PARME

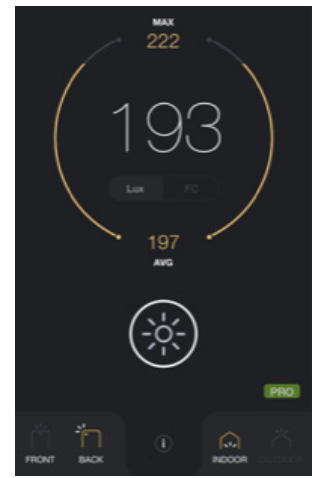

Figure 2: The application on a phone when pointed at a desk lamp.

measurements with the phone being pointed at a lamp rather than the LEctenna.

The PARME only requires easily attainable household items to construct. The main items consist of thick bubble tea straws, cardboard, and glue. The first part to build is the shaft and the area that supports the back of the phone. First a hole was cut through the center of a piece of cardboard, just large enough for the bubble tea straw to slide into, the dimensions being $3.20 \times 3.20 \mathrm{~cm}$. The diameter of the straw was about $1 \mathrm{~cm}$ long. The straw that served as the shaft was $3 \mathrm{~cm}$ in length. Then, two straws were measured to a length of $3.20 \mathrm{~cm}$ and placed them on either side of the shaft for support. Additionally, a smaller piece of straw, with a length long enough to reach the shaft and the outer edge of the cardboard, was attached. Finally, glue was to secure all the pieces on the cardboard. The finished piece is shown in Figure 4.

Next is the piece that allows the device to be attached to the phone. A piece of cardboard was cut to a width of $1 \mathrm{~cm}$ and a length between $3-5 \mathrm{~cm}$ (depending on thickness of phone) and glued flat on top of the center straw piece in Figure 4. Then, at the end not connected to the straws, the cardboard was bent downwards at a 90-degree angle and it was glued in place with the help of other small cardboard pieces. The bend location finished piece is shown in Figure 4. Multiple layers of cardboard can be added for stability. Once the glue dries, this next part is determined by phone size and may be slightly different for different phones. The phone was held so that the camera was aimed inside the shaft of the device. Then, small pieces of cardboard were added above and besides the phone so that the device attaches to the phone without any outside support.

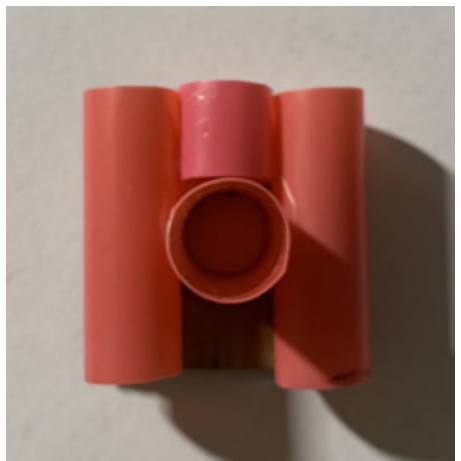

Figure 3: The shaft and the arrangement of the bubble tea straws used for support.

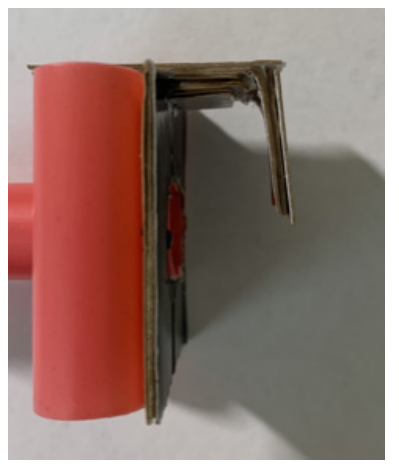

Figure 4: The piece that allows a phone to be attached to the PARME.

The final piece held the LEctenna in place. A piece of straw that was cut to $3.5 \mathrm{~cm}$ in length was then cut horizontally in half on one end, so that the inside of the straw is exposed. At the exposed side, two slits, on either side, were cut so the LEctenna could be placed. Next, the other half was cut parallel down the middle of the straw so that the radius of the straw can be adjusted to a size that will be able to fit in the shaft. Again, a tighter fit was preferred over a looser fit. Some tape was used to secure the appropriate size. The finished piece is shown in Figure 5.The completed PARME device is shown in

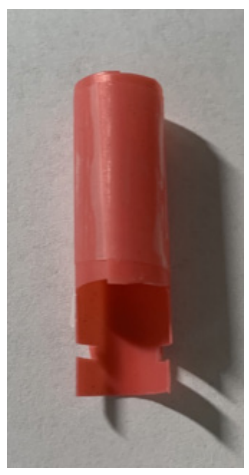

Figure 5: The piece that holds the LEctenna in place. 


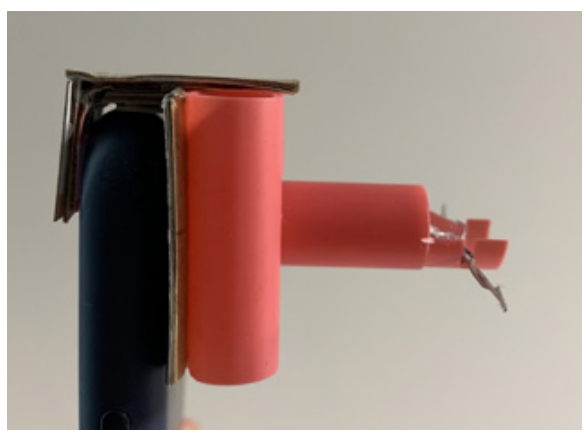

Figure 6: The PARME attached to the phone.

Figure 6. The piece in Figure 5 is slid inside the shaft of the piece shown in Figure 3. Figure 6 shows how the PARME should be attached to the phone. It is important to ensure before collecting data that the PARME is correctly placed over the phone camera. If the PARME ends up shifting around when in use, small pieces of cardboard can be added to increase stability.

\section{- Results and Discussion \\ The LEctenna:}

The rectifying antenna is called the rectenna. A rectenna is a type of receiving antenna that converts electromagnetic energy, such as radio or microwaves, into direct current that can be used to power everyday objects. ${ }^{3}$ The LEctenna is composed of two components: an LED and a Schottky diode, whose leads form the antenna. An image of the LEctenna can be seen in Figure 7. A Schottky diode is generally used because it has the lowest voltage drop and the fastest switching speed. ${ }^{4}$ The diode only allows current flow in one direction, which occurs when it is forward-biased. The voltage across a forward-biased light emitting diode results in a current that will illuminate the LED. ${ }^{5}$ When held up to different objects, such as Wi-Fi router antennas and microwave ovens, the LEctenna will have varying levels of brightness and lux values because the different objects transmit waves differently. Both red and green LEDs were used to make LEctennas to compare patterns and make assumptions.

\section{Phone Attachable RF Meter Enhancer (PARME):}

In an attempt to collect more concrete measurements, a downloadable application on a smart phone was used to measure the amount of lux that the LEctenna emitted. The name of the application being Lux Light Meter Pro. ${ }^{6}$ Although the application works well and is fairly accurate, the flashing of the LEctenna causes the emitted light to only be partially cap-

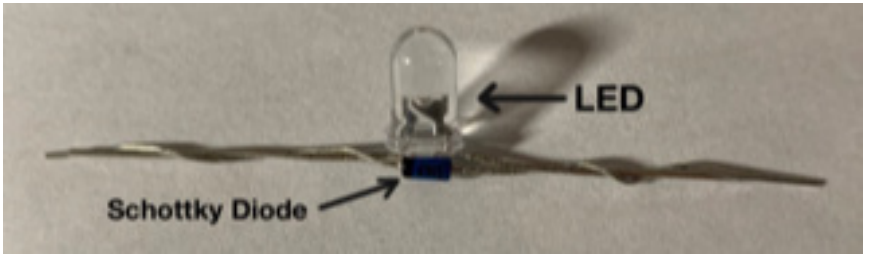

Figure 5: The piece that holds the LEctenna in place.

tured, which results in some error margins. After some poor attempts to measure lux, it became clear that a better way for the light to be focused directly at the smart phone camera was needed. Then, a device, which can be attached over a phone camera was created, allowing the light from the LEctenna to be directed at the phone camera for more conclusive measurements. This device was only tested with an iPhone XS but can be altered to be compatible with other phone designs. Figure 8 shows the completed PARME, fitted to the dimensions of an iPhone XS.

\section{Brightness Scale:}

Defining a brightness scale in more concrete terms is important, as describing the brightness of an LED has the potential for ambiguity. This brightness scale measures the voltage of the LEctenna in different situations and categorizes the brightness into 4 levels. As the data for the distinct brightness levels was collected, as shown in Table 1 , a digital multimeter with alligator clips was connected to the LEctenna, which in-

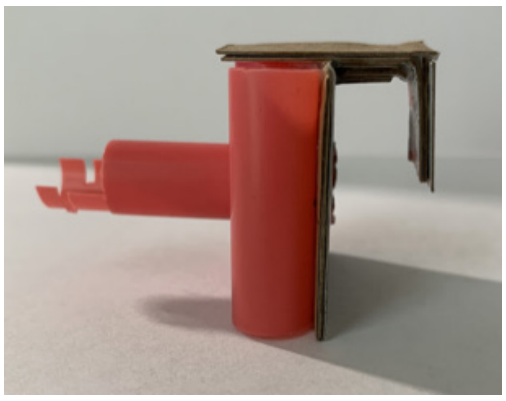

Figure 8: The finished PARME device.

creased resistance in the circuit. In order for the voltage of the LEctenna to reach a specific amount, cardboard that is covered in aluminum foil was used to increase the brightness. These cardboard pieces are shown in Appendix A.

The first brightness level is determined as just a speck of light. The LEctenna was held 5 to $7 \mathrm{~cm}$ away from the $\mathrm{Wi}-\mathrm{Fi}$ antenna and slowly slid the LEctenna closer until a speck of light appeared. Then the Lectenna was slid towards the antenna until distinct changes in brightness was observed, which led to the development of more brightness levels. In Figures 9 and 10 , images of level one brightness are shown for both different color LEctennas. Any voltage that was less than the minimum voltage for level 1 for either $\mathrm{AC}$ or DC was too dim to be observed or no light was emitted from the LED.

From level 2 to level 4, the distinction between levels is visually more subtle. The second brightness level is noticeably brighter than level 1, but still not incredibly bright. Figures 11 and 12 show generally what a level 2 brightness looks like compared to level 1.

Table 1: The 4 levels of brightness with the corresponding voltage values.

\begin{tabular}{|l|l|l|l|l|}
\hline & \multicolumn{2}{|c}{ Green LED } & \multicolumn{2}{c}{ Red LED } \\
Voltage (volts) & DC Voltage & AC Voltage & DC Voltage & AC Voltage \\
\hline Levels & & & & \\
\hline Level 1 & $0.15-0.30$ & $0.20-0.30$ & $0.10-0.20$ & $0.10-0.20$ \\
\hline Level 2 & $0.35-0.50$ & $0.30-0.60$ & $0.25-0.36$ & $0.36-0.55$ \\
\hline Level 3 & $0.60-0.75$ & $0.70-1.00$ & $0.40-0.55$ & $0.65-0.90$ \\
\hline Level 4 & $0.75-1.10$ & $1.00-1.50$ & $0.60-0.75$ & $1.00-1.30$ \\
\hline
\end{tabular}




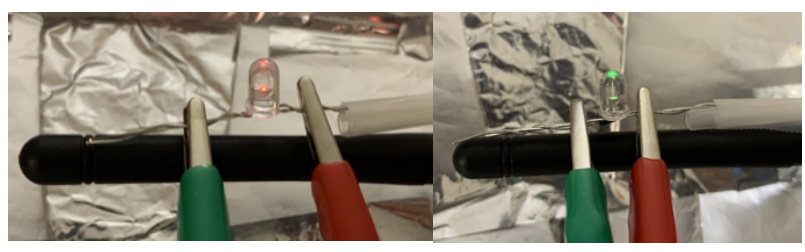

Figures 9 and 10: The appearance of level 1 brightness for the two different colored LEctennas.

The third brightness level is brighter than the second level and appears fuller than earlier levels. Figures 13 and 14 can be compared with prior figures to compare the brightness.

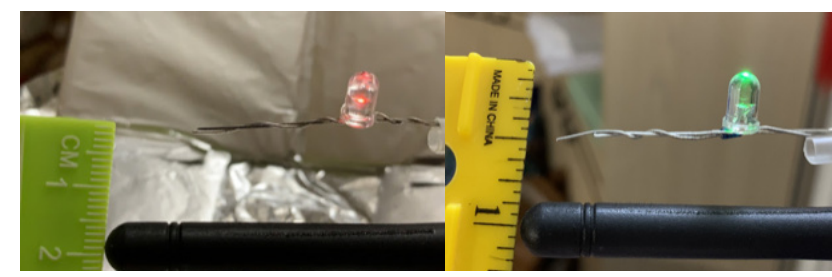

Figures 11 and 12: The appearance of level 2 brightness.

Although the voltages were greater for level 3, the pulsing of the LEctenna increased and, as a result, the brightness varied between extremes more rapidly.

The fourth and the highest tier in this brightness scale can be described as noticeably the brightest and fullest in light.

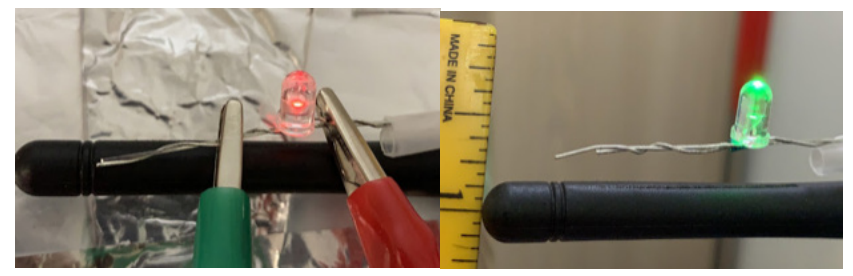

Figures 13 and 14: The appearance of level 3 brightness.

Overall, the voltage for the red LED LEctenna is generally lower than the green LED LEctenna, but as seen in Figures 15 and 16 , their brightness' seem similar.

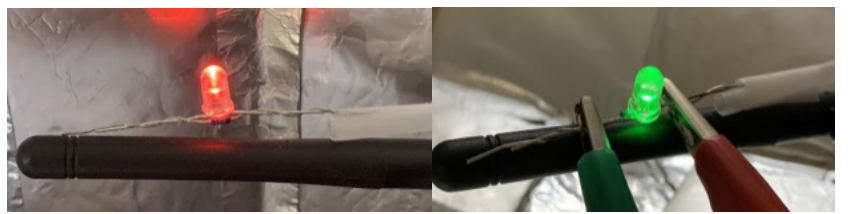

Figures 15 and 16: The appearance of level 4 brightness.

\section{Brightness and Waves:}

From prior sections and Appendix B, we can understand the effects the diode has on the LED and how the two components work as a whole. Each added Schottky diode corresponds with a voltage drop of 0.2 to 0.3 volts. Next, the behavior of the LEctenna, as it is held next to the WiFi antenna, was examined.

Initially, as the LEctenna was held up to the WiFi router antenna at distance zero with no alligator clips attached, the brightness varied around levels 2 to 4 , depending on which color LED was used. The flashing of the LEctenna makes it more difficult to record completely accurate information, hence the error margins in the Figure 18 that appears qualitatively to be approximately 10 percent. The intermittent delivery of network packets is presumed to be the cause of the LEctenna's sporadic flashing. Network packets are individual units that carry information from an origin to a destination in a network. ${ }^{7}$ When a message is sent, it is broken up into packets and sent to its destination, where it is then rearranged and reassembled. When more packets are sent over a specific unit of time, the LED's brightness increases, due to the larger amount of energy delivered. The amplitude of the wavelength correlates to the amount of energy that the packet carries. Larger amplitude means greater energy per packet. Additionally, many things, such as physical objects, other wireless appliances, and kitchen appliances, interfere with the Wi-Fi signal and can cause slow network speeds, pauses in connection, and poor signal strength. ${ }^{8}$

Another possible explanation for the pulsing of the LEctenna is the peaks and troughs of the waves. The different peaks and troughs correspond to how electrical charge is distributed through the wave. Since the diode only allows current flow in one direction, the positive amplitude portion of the wave will light up the LEctenna, but the negative amplitude portion will not. In Figure 17, a visual representation of a wave can be seen. The portions with a dotted line below the yellow line will cause the LEctenna to shut off. The solid lines represent the portion of the wave that turns on the LEctenna.

In Figure 18, a graph was created of how the voltages of

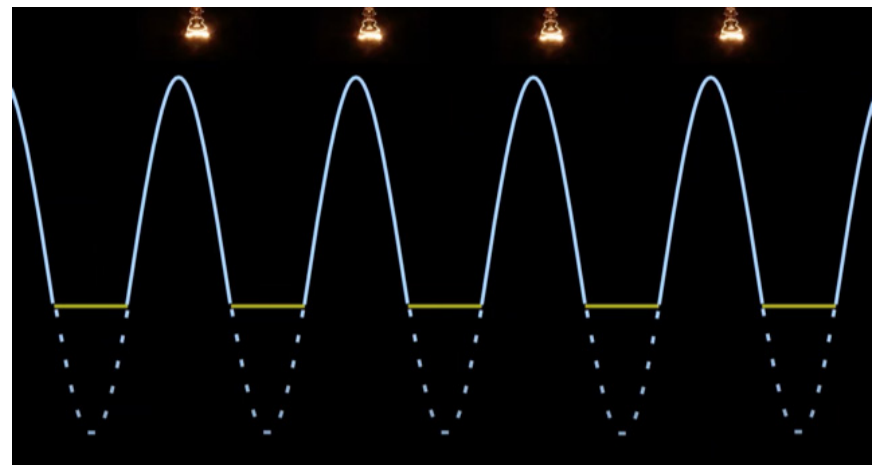

Figure 17: This is a representation of the different sections of the wave as it is rectified by the LEctenna. ${ }^{9}$

the different LEctennas and diodes vary as they are held at different distances from the center axis of the WiFi antenna. The forward voltage of the green LED is greater at around 3.2 to 3.8 volts compared with the red LED's forward voltage of 1.8 to 2.2 volts. When comparing the brightness of the two LEctennas, the green LED LEctenna appeared to be brighter than the red LED LEctenna. Additionally, humans perceive green light as being brighter than red light. ${ }^{10}$ When collecting the data, the Schottky diode behaved very interestingly. Out of the three devices, the diode had the lowest average voltage. Occasionally, the Schottky diode's voltage would become very low at around 0.01 for a few seconds, but this data was mostly disregarded.

As suspected, the voltage of the LEctenna varies when it is held up to different objects. For example, when the LEctenna 
is held next to a microwave oven door crack, the voltage of the LEctenna is higher as a result of the microwaves being steadily delivered, rather than in small packets. When held directly on the crack of the door, the voltage of the green LED LEctenna was around 2.0 volts, which is about twice the voltage of the same LEctenna as it is held against the WiFi router antenna. The microwave oven generates more power than a WiFi router, which causes the LEctenna to be brighter.

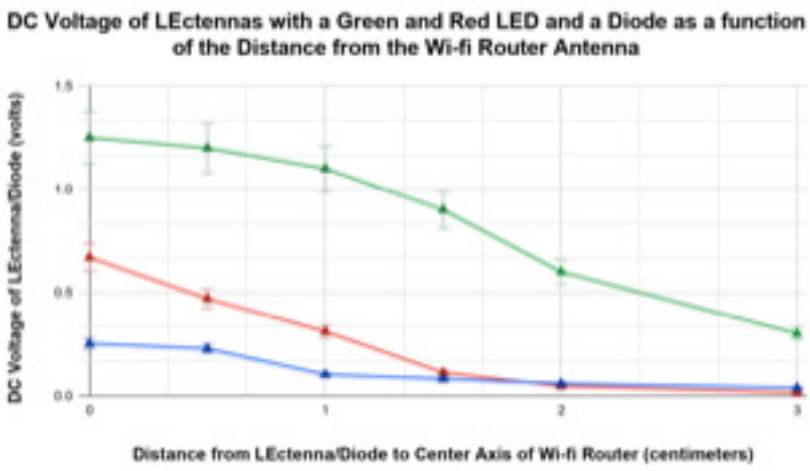

Figure 18: The measured DC voltage of LEctennas with green and red LEDs and a Schottky diode alone when measured at various distances from the center axis of the $\mathrm{Wi}-\mathrm{Fi}$ router antenna.

Figure 19 shows the data that was recorded using the Lux Light Meter Pro application and the PARME. The LEctenna was held at different distances from the center axis of the $\mathrm{Wi}-\mathrm{Fi}$ router antenna and the average and maximum lux for multiple trials were recorded. The values for average and maximum lux were taken directly from the application over a 10 to $20 \mathrm{sec}-$ ond period. The periods were dependent on the behavior of the LEctenna. If the brightness was dimmer, the period would be extended in order to get more accurate results. For the values of average lux, the red LED LEctenna had overall higher values than the green LED LEctenna. Interestingly, for the maximum lux, the green LED LEctenna had greater values than the red LED LEctenna. This data could possibly be skewed by the inaccuracy of the Lux Light Meter app. Although the lux values varied between the different color LEctennas, at a distance of zero centimeters, both LEctennas emitted a level 4 brightness. However, the average values for a distance of 2 and 3 centimeters were very close to the value of zero, and as a result, the values were rounded to zero for simplification. Additionally, the maximum values for distances 2 and 3 centimeters were also around a value of 10 , so similarly, they were rounded to 10 lux for simplification. The larger margins of error account for the inaccuracy of the lux meter caused by the pulsing of the LEctenna.

In both Figures 18 and 19, a general pattern is shown. As the LEctenna is moved away from the $\mathrm{Wi}$-Fi router antenna, the lux and voltage decrease. Because of the way that antenna signals work, the power density of the signal decreases as the LEctenna is moved farther from the antenna. ${ }^{8}$

A separate experiment was conducted to determine how the range of the LEctenna could be increased. Simple household objects like tin foil and cardboard can be used to construct reflectors that can be aimed at the LEctenna and $\mathrm{Wi}$-Fi router to increase range. More on this experiment is shown in Appendix A.

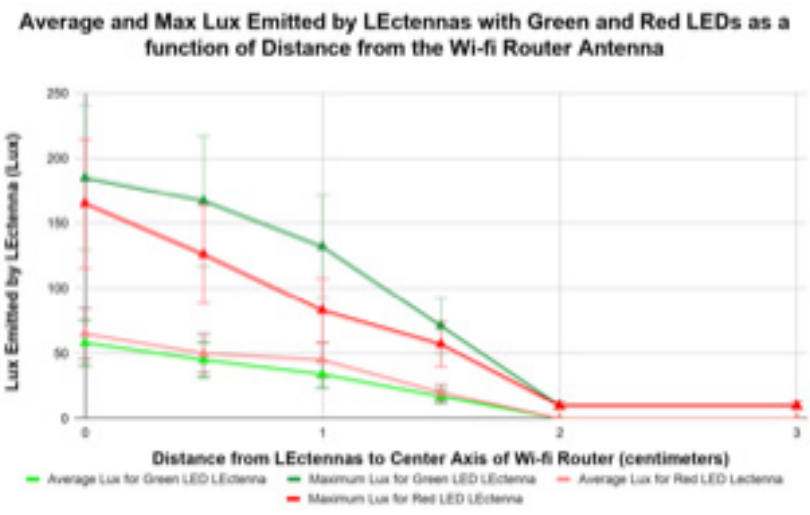

Figure 19: This line chart shows the average and maximum lux values of the LEctennas with a green and red LED when measured from the center axis of the $\mathrm{WiFi}$ router.

\section{Conclusion}

In conclusion, with the LEctenna as a visual representation, the voltage and lux recorded by the PARME allow us to understand the presence and relative strength of microwaves found in the home. The flashing of the LEctenna shows that there are individual packets being rapidly sent through the $\mathrm{Wi}-\mathrm{Fi}$ network. Furthermore, the different voltage and lux values for the different color LEctennas reveal that color of the LED is a determining factor for perceived brightness. These conclusions can be drawn from the results that conform to the expected antenna characteristics. From the behavior of the LEctenna, general trends about increased distance and weakened power can be related and used in power beaming. In addition, the LEctenna can also be used as an example to show the public that the process of power beaming could be a safe and efficient way to transmit power to specific locations on Earth.

\section{Acknowledgement}

I would like to express my deepest gratitude to Dr. Paul Jaffe of the Naval Research Laboratory for assisting me and sharing his deep knowledge and experience in every stage of this research project.

\section{References}

1. Space Solar Power. https://space.nss.org/space-solarpower/ (accessed Jul 24, 2020).

2. Wood, D. Space-Based Solar Power

https://www.energy.gov/articles/space-based-solar-power

(accessed Jul 20, 2020).

3. Aboualalaa, M., \& Elsadek, H. (2019,

October 29). Rectenna Systems for RF Energy

Harvesting and Wireless Power Transfer. Retrieved

August 17, 2020, from

https://www.intechopen.com/books/recent-wirelesspower-transfer-technologies/rectenna-systems-for-rfenergy-harvesting-and-wireless-power-transfer

4. Schottky Diode Working and Its Applications. https://www.elprocus.com/schottky-diode-working-andapplications/ (accessed Aug 3, 2020).

5. Introduction to Diodes And Rectifiers. 
https://www.allaboutcircuits.com/technical-articles/ (accessed Aug 2, 2020).

6. Polyanskaya, M. Lux Light Meter Pro; Measure Luminosity in Lux \& FC, 2017.

7. What is a packet? https://beambox.com/townsquare/ what-s-the-difference-between-2-4ghz-and-5ghz-wifi (accessed Aug 1,2020).

8. How to troubleshoot $\mathrm{Wi}$-Fi Interference with NetSpot https://www.netspotapp.com/wifi-interference. html (accessed Jul 30, 2020).

9. USNRL. LEctenna on ISS; Youtube: USA, 2020.

https://www.youtube.com/watch?v=zo7w0D6vz5g

10. Visible Light and the Eye's Response https:/www.physicsclassroom.com/class/light/Lesson-2/ Visible-Light-and-the-Eye-s-Response (accessed Aug 1, 2020).

\section{- Appendix A: Maximizing Range}

For maximizing the range of the LEctenna, three different cardboard pieces covered with aluminum foil were used. The first piece (piece $A$ ) is one that was placed on an object about 5-6 centimeters below the LEctenna and the router antenna. In Figure 20, piece $\mathrm{A}$ is shown. To start, the 3 larger cardboard aluminum foil pieces, identical to each other with dimensions of $7 \times 10 \mathrm{~cm}$, were connected at the longer side. One piece is laid flat on the table while the other two pieces are angled above the horizon. Figure 21 shows how the pieces were angled. This allows waves to be reflected off the aluminum foil and towards the LEctenna. Next, 2 smaller pieces of cardboard aluminum foil, with dimensions of $3 \times 3.5 \mathrm{~cm}$, were taped to the middle piece of the three pieces, directly above the shorter side, also at similar angles as the larger pieces were placed. Finally, aluminum foil was used to connect the edges of the smaller pieces to the edges of the larger pieces. In the pictures, the creases were covered with more aluminum foil, but it is unclear if it changed the effectiveness.

The second piece (piece B) is the smallest piece of cardboard covered with aluminum foil. Figure 22 shows the dimensions and the shape of the piece B.

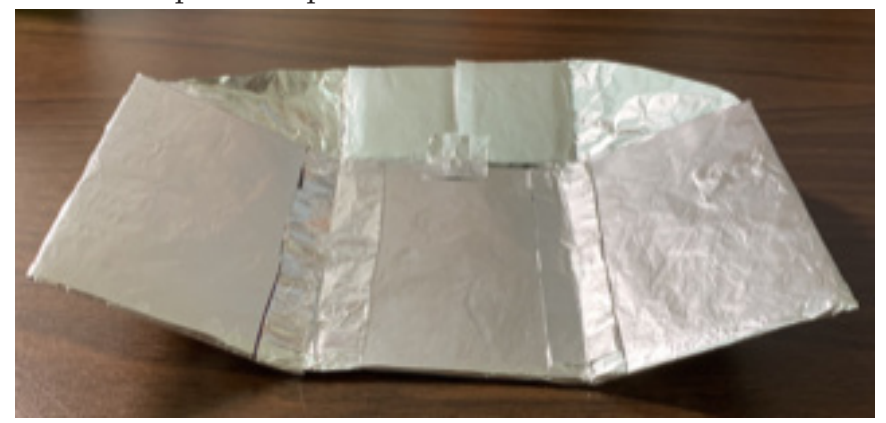

Figure 20: This is the completed piece A.

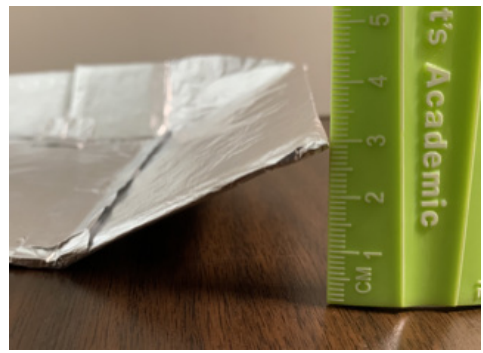

Figure 21: The angle of the pieces.
The third piece (piece C) consisted of three smaller pieces taped together. The largest piece had dimensions of $15.25 \mathrm{x}$ $7.60 \mathrm{~cm}$ and the smaller pieces were identical to each other

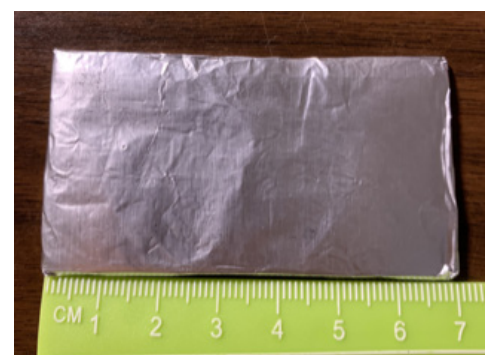

Figure 22: The dimensions $(7 \times 3.8 \mathrm{~cm})$ of the second piece of cardboard covered foil.

with dimensions of $6.35 \times 5.0 \mathrm{~cm}$. As the largest piece was horizontally laid flat on the table, the two smaller pieces were taped at the top of the longer side, not beside the piece. Each piece was taped at the farthest ends of the larger piece, with a space between the two smaller pieces. Then as the larger piece was bent in the middle, the space between the two smaller pieces was connected with aluminum foil. These creases were covered with aluminum foil. For clarification, see Figures 23 and 24.

When experimenting, the method that was found to be effective was to hold pieces $\mathrm{B}$ and $\mathrm{C}$ up to the LEctenna and antenna, while piece $\mathrm{A}$ is placed beneath all the objects. Figure

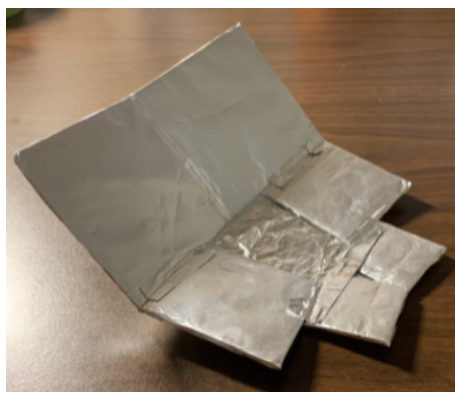

Figure 23: The completed third piece for maximizing range.

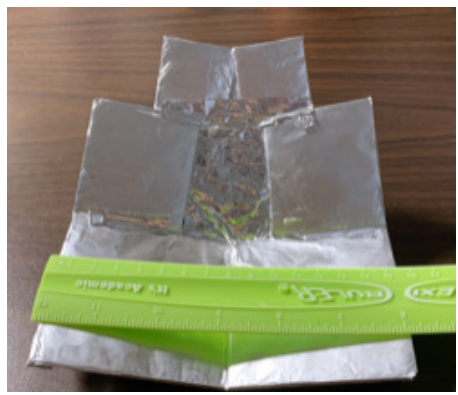

Figure 24: The dimensions of the third piece.

25 shows how the pieces were set up. To better maximize the brightness results, piece $\mathrm{B}$ is held closer to the $\mathrm{Wi}-\mathrm{Fi}$ router and piece $\mathrm{C}$ is held closer to the LEctenna. It is important to note that the aluminum foil part of piece $\mathrm{C}$ is held adjacent to the LEctenna. Piece B should be aimed at the LEctenna or 
the aluminum foil behind the LEctenna. Varying the direction that the pieces are facing and the positions that the LEctenna is held at besides the antenna will help to maximize brightness. To continue the experiment, the LEctenna was moved away from the router antenna while the cardboard aluminum foil pieces were moved accordingly and used the same method to determine the farthest distance from the router that the LEctenna would light up at. The farthest that was able to be reach was around $12.7 \mathrm{~cm}$ with a voltage ranging between 0.5 to 1 volt.

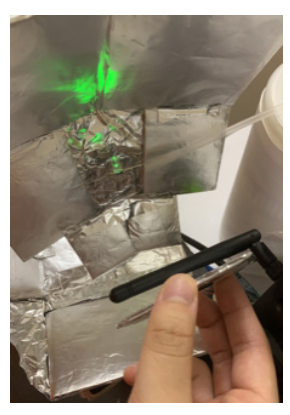

Figure 25: The set up the cardboard foil.

\section{Appendix B: Multiple Diodes}

When making the LEctenna with multiple Schottky diodes, two methods were used. The first method is similar to the one for making a simple LEctenna. First a simple LEctenna was used. Then the diode was wrapped around the LED 3-4 times while making sure that the ends of the leads were straight. Then, the other diode was wrapped it 3-4 times around the LEctenna that was just made. The second method that was used was, first, the diodes were wrapped around each other 3-4 times. Then, the double diode was wrapped around the LED. In both methods, the leads were twisted in a way that would maximize the amount of contact the two leads had with each other.

When using multiple diodes, it was clear that although both one diode LEctennas and two diode LEctennas lit up, the LEctennas with only one diode lit up brighter. When comparing the data that was collected from the two different types of LEctennas with green LEDs, the LEctenna with one diode had a DC voltage of around 1 volt while the LEctenna with two diodes had a DC voltage of around 0.8 to 0.85 volts. However, even though they have similar brightness levels, the behaviors of the two LEctennas were different. With only one diode, the pulsing of the LED fluctuated between brightness levels at a faster pace. With 2 or 3 diodes, even though the light was dimmer, it was emitted more steadily.

The method that was used to wrap the two diode LEctennas did not have much effect on the overall performance of the LEctenna. Whether method one or method two was used, the determining factor is the overall amount that the leads contacted each other.

One possible reason that the LEctenna with two diodes had a lower voltage is that the added diode caused the voltage to drop more. Through experimentation, one can conclude that an added diode resulted in a voltage drop from around 0.20 to 0.30 volts. This pattern was displayed with the LEctenna with the green LED as well as with the LEctenna with a red LED. The voltage drop of the green LED alone was around 3 volts and the red LED alone was around 2 volts. Although with one diode, the voltage dropped more than around 0.20 volts, but with every added diode this trend occurred. With one diode, the voltage of this LEctenna was around 0.80 to 0.90 volts. With two diodes, the voltage dropped to around 0.60 to 0.65 , which is around a 0.20 to 0.30 drop.

\section{Author}

Michelle Yu, a junior at Thomas S. Wootton High School, is interested in many aspects of STEM, such as biology, biochemistry, and engineering. With high hopes to influence and help others around her, she wants to pursue either medicine or biomedical sciences. 